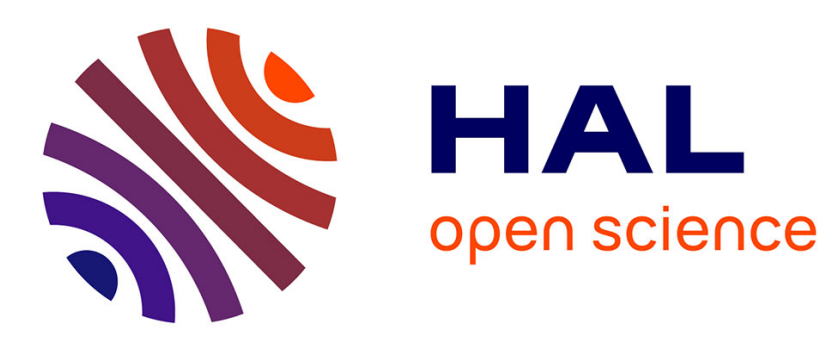

\title{
Pulse propagation near zero group velocity dispersion in a femtosecond dye laser
}

François Salin, Philippe Grangier, Patrick Georges, Alain Brun

\section{To cite this version:}

François Salin, Philippe Grangier, Patrick Georges, Alain Brun. Pulse propagation near zero group velocity dispersion in a femtosecond dye laser. Optics Letters, 1990, 15 (23), pp.1374-1376. hal00691668

\section{HAL Id: hal-00691668 \\ https://hal-iogs.archives-ouvertes.fr/hal-00691668}

Submitted on 26 Apr 2012

HAL is a multi-disciplinary open access archive for the deposit and dissemination of scientific research documents, whether they are published or not. The documents may come from teaching and research institutions in France or abroad, or from public or private research centers.
L'archive ouverte pluridisciplinaire HAL, est destinée au dépôt et à la diffusion de documents scientifiques de niveau recherche, publiés ou non, émanant des établissements d'enseignement et de recherche français ou étrangers, des laboratoires publics ou privés. 


\title{
Pulse propagation near zero group-velocity dispersion in a femtosecond dye laser
}

\author{
F. Salin, P. Grangier, P. Georges, and A. Brun \\ Institut d'Optique Théorique et Appliquée, Unité Associée au Centre National de la Recherche Scientifique, U.A. 14, Université Paris-Sud, \\ B.P. 147, 91403 Orsay Cédex, France
}

Received June 19, 1990; accepted October 8, 1990

\begin{abstract}
The propagation of femtosecond pulses in a colliding-pulse mode-locked dye laser near zero group-velocity dispersion is studied. The pulse spectrum is shown to exhibit a double-peak structure. This structure and its dependence on the intracavity dispersion can be explained by nonlinear pulse propagation near zero dispersion. A value for the third-order dispersion of the laser cavity is deduced and is found to be predominant for pulses shorter than 50 fsec.
\end{abstract}

Femtosecond pulses have been obtained by using different types of laser, resulting in pulses shorter than 30 fsec. Among these techniques, one of the simplest and most powerful is the colliding-pulse mode-locked (CPM) laser. Since its introduction in $1981,{ }^{1}$ this method has significantly evolved. It became obvious that pulses were formed not only by gain and loss saturation but also as a result of nonlinear effects. It was shown that the shortest pulses are obtained when the intracavity group-velocity dispersion (GVD) and self-phase modulation (SPM) are balanced. ${ }^{2}$ This led to the concept of soliton shaping. ${ }^{2-5}$ The experimental proof of the pulses' solitonlike character was obtained recently.6,7 In order to obtain this solitonlike propagation, the cavity GVD has to be perfectly controlled. Several authors have studied both experimentally and theoretically the influence of GVD on the laser characteristics. By comparing the results of a numerical model and the autocorrelation functions of the pulses produced by a spectrally windowed CMP laser, Avramopoulos et al. have shown that the shortest pulses are obtained for small negative GVD. ${ }^{4}$ Recently we experimentally measured the GVD of our CPM laser by using $N=2$ solitonlike pulse properties and found that the optimum GVD is negative and extremely small. ${ }^{8}$ All these results show that the higher-order dispersion terms have to be taken into account. Recently de Barros et al. showed theoretically that the third-order term could have an influence on the pulse characteristics. ${ }^{9}$ In this Letter we report an experimental study on the characteristics of pulses produced by a CPM laser operating near the zero of the second-order dispersion and we show that the experimental results are relatively well explained if one takes the third-order term into account.

The experiment was conducted using a classical sixmirror CPM laser including four silica prisms. ${ }^{10}$ The dyes used were Rhodamine 6G and DODCI dissolved in ethylene glycol. All the mirrors were single stack and coated for $100 \%$ reflectivity at normal incidence at $620 \mathrm{~nm}$. The output coupler had a transmission of $1 \%$. Starting from the alignment that gives the shortest pulses (typically $60 \mathrm{fsec}$, although we have obtained 31-fsec pulses with this cavity), we increased the DODCI jet thickness by translating the jet vertically. The estimated thickness is then above $50 \mu \mathrm{m}$. The pulse width increased to approximately $80 \mathrm{fsec}$, while the wavelength shifted to the red. The exact effect of translating the absorber jet is not perfectly understood. It could increase the effect of SPM and reduce the shaping mechanism due to the absorber and gain. When one of the prisms is translated, introducing a little more glass in the cavity than is needed to get the shortest pulses, a complicated spectral and temporal behavior is observed. A second peak appears in the pulse spectrum at a wavelength that depends on the intracavity GVD. When the spectrum presents two peaks the autocorrelation trace evolves from the usual clean near-sech ${ }^{2}$ shape to a complicated multiplepeaked shape depending on the orientation of the autocorrelator doubling crystal (i.e., on the wavelength of the doubled signal). The KDP doubling crystal was $100 \mu \mathrm{m}$ thick, and its acceptance bandwidth was much narrower than the 20 -nm bandwidth of the pulses. We found that the main peak of the spectrum corresponded to a stable and clean pulse. Figure 1 shows the evolution of the pulse spectrum as a function of intracavity GVD variation. A second spectral peak is clearly visible on the right, and its position changes until it merges with the principal part of the spectrum. No modulation of the pulse train envelope reminiscent of high-order solitonlike pulses ${ }^{6,7}$ was observed until the two peaks merged. Figure 2 gives the position of the two peaks as a function of the prism position. Obviously this position cannot be accurately measured for the first curve. When more than two spectral peaks can be seen in Fig. 1, only the two wavelengths with the highest peaks have been considered. Such a splitting of a pulse spectrum has already been observed in an optical fiber ${ }^{11}$ and in a CPM laser. ${ }^{12}$ It has been attributed to the third-order dispersion when the pulse propagates near the zero second-order dispersion of the fiber or the laser and has been studied by several authors. ${ }^{13-15}$

When a pulse is launched near zero dispersion, a part of its spectrum lies in the positive dispersion 


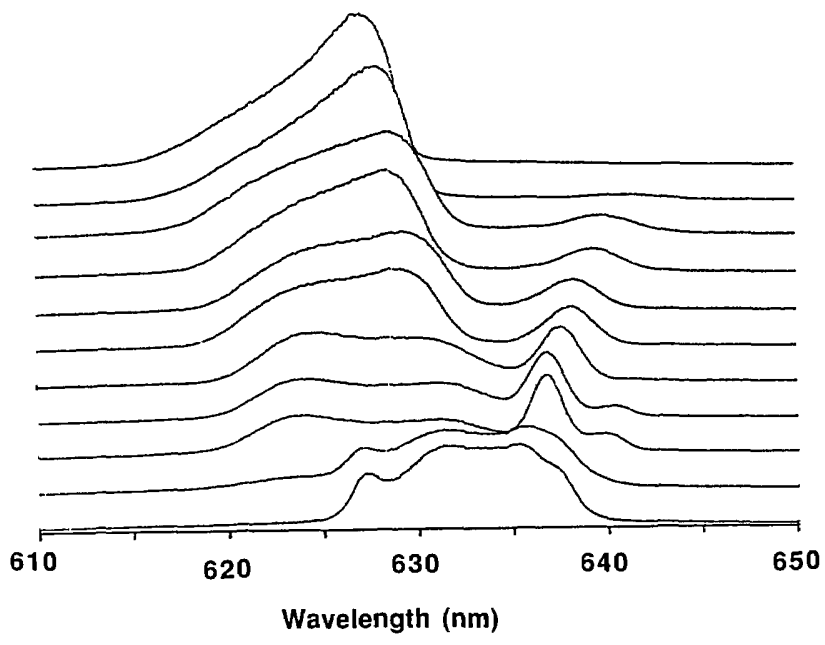

Fig. 1. Evolution of the pulse spectrum with intracavity GVD. Positive GVD is added from the top to the bottom of the figure.

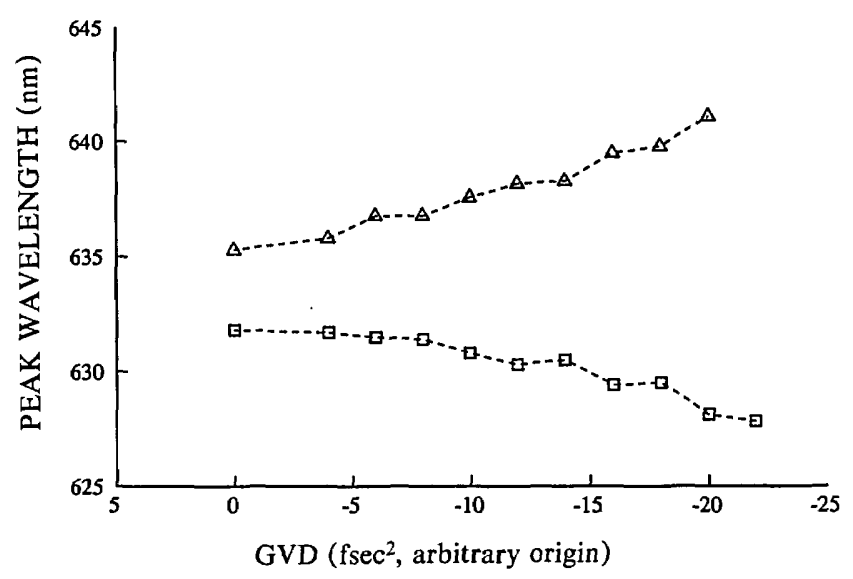

Fig. 2. Evolution of the soliton ( $\square$ ) and spectral resonance $(\Delta)$ wavelengths as a function of the intracavity group-velocity variation.

region and the rest of the energy is in the negative GVD region. This last part of the pulse can evolve into a soliton, while, in the positive GVD region, the SPM is not balanced by the GVD, and the energy spreads in time. Nevertheless, if one takes into account third-order dispersion, there is a wavelength in the positive GVD region where the group velocity is the same as the main pulse group velocity. A part of the energy is then trapped in the pulse and leads to a spectral resonance. The wavelength of this resonance depends on the third-order dispersion value and conversely can be used to measure this value. Note that the same phenomenon is observed for the propagation of soliton ${ }^{14,15}$ or nonsoliton ${ }^{13}$ pulses in fibers. In that case the resonance does not correspond to the frequency at which the group velocity is equal to that of the main pulse but to the frequency at which the time derivatives in the nonlinear Schrödinger equation describing the pulse propagation cancel.

Assuming that, in order to be stable, the different parts of the spectrum have to travel at the same speed, the exact position of the spectral resonance can be obtained in a simple manner. Let us write the group velocity $V_{0}$ at a frequency $\omega_{0}$,

$$
V_{0}=\left[\left(\frac{\mathrm{d} k}{\mathrm{~d} \omega}\right)_{\omega=\omega_{0}}\right]^{-1}=k_{0}^{\prime-1},
$$

where $k$ is the wave number. We can obtain the group velocity $V$ at a wavelength $\omega$ near $\omega_{0}$ by using a secondorder expansion of the group velocity,

$$
V=V_{0}+\left.\frac{\mathrm{d} V}{\mathrm{~d} \omega}\right|_{\omega=\omega_{0}}\left(\omega-\omega_{0}\right)+\left.\frac{1}{2} \frac{\mathrm{d}^{2} V}{\mathrm{~d} \omega^{2}}\right|_{\omega=\omega_{0}}\left(\omega-\omega_{0}\right)^{2} .
$$

Introducing the second- and third-order GVD,

$$
k_{0}^{\prime \prime}=\left.\frac{\mathrm{d}^{2} k}{\mathrm{~d} \omega^{2}}\right|_{\omega=\omega_{0}} \text { and } \quad k_{0}^{\prime \prime \prime}=\left.\frac{\mathrm{d}^{3} k}{\mathrm{~d} \omega^{3}}\right|_{\omega=\omega_{0}}
$$

at the wavelength $\omega_{0}$ and assuming that $k^{\prime \prime 2} \ll k^{\prime \prime \prime}$, we obtain

$$
V=V_{0}-\frac{k_{0}^{\prime \prime}}{k_{0}{ }^{2}}\left(\omega-\omega_{0}\right)-\frac{1}{2} \frac{k_{0}^{\prime \prime \prime}}{k_{0}{ }^{2}}\left(\omega-\omega_{0}\right)^{2} .
$$

We can now look for two wavelengths $\omega_{1}$ and $\omega_{2}$ that have the same velocity. By using Eq. (3), this leads to

$$
\left(\omega_{1}-\omega_{2}\right)\left[k_{0}{ }^{\prime \prime}+1 / 2^{2} k_{0}^{\prime \prime \prime}\left(\omega_{1}+\omega_{2}-2 \omega_{0}\right)\right]=0 .
$$

In order to check the fit between Eq. (4) and the experiment, we have plotted in Fig. 3 the variation of the quantity $\omega_{1}+\omega_{2}-2 \omega_{0}$ obtained from Fig. 2 versus the variation of $k_{0}{ }^{\prime \prime}$. The frequency $\omega_{0}$ was arbitrarily chosen as the mid-frequency between the two leftmost points of Fig. 2. The experimental points are close to the straight line predicted by Eq. (4). A leastsquares fit gives a slope of $-1800 \mathrm{fsec}^{3}$, leading to a third-order intracavity dispersion of $-3600 \mathrm{fsec}^{3}$. We note that this value is negative as expected if the major source of third-order dispersion comes from the prisms. We can also use Eq. (4) to estimate the second-order dispersion of the cavity. Using the spectral shift $\left(\omega_{1}+\omega_{2}-2 \omega_{0}\right)$ corresponding to the top curve of Fig. 1, we obtained $k_{0}{ }^{\prime \prime}=-60 \mathrm{fsec}^{2}$ for this particular prism position. In a recent experiment ${ }^{8}$ we obtained

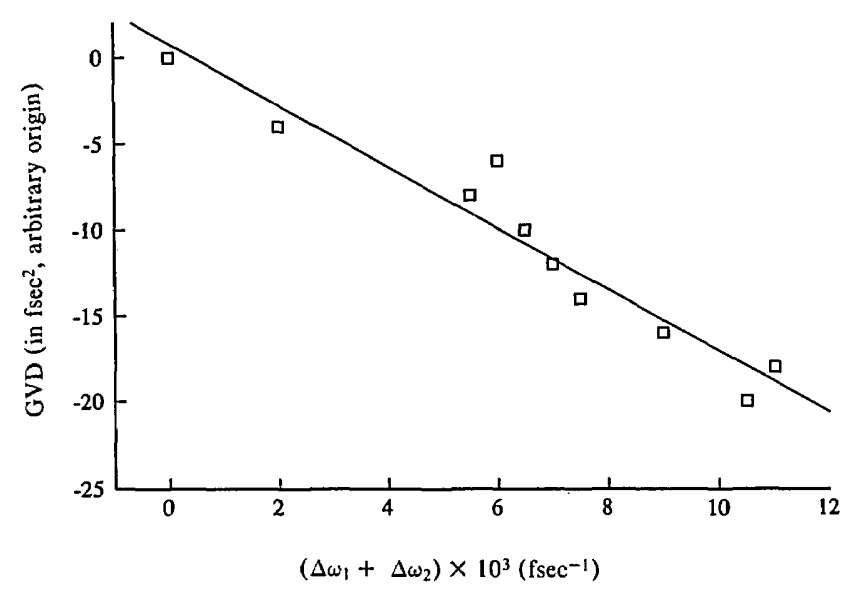

Fig. 3. Sum of the relative variations of the two wavelengths plotted in Fig. 2 versus GVD. The straight line is a calculation using Eq. (4). 
an estimation of the cavity GVD of approximately -20 fsec $^{2}$ for a 40 -fsec pulse. From previous results ${ }^{4,16}$ we can deduce values in the range of -20 to $-100 \mathrm{fsec}^{2}$ for 60 - to 80 -fsec pulses. All these values fall in the same range and seem to indicate that the cavity GVD is of the order of $-50 \mathrm{fsec}^{2}$. In order to compare the influence of the second-and third-order dispersion we have computed the value of the term $1 / 3 \phi^{\prime \prime \prime} \Delta \omega$ with a $\Delta \omega$ corresponding to a 50 -fsec transform-limited pulse. The result is approximately $-50 \mathrm{fsec}^{2}$. This value is equal to or even greater than the GVD of femtosecond lasers generating 50 -fsec pulses $4,8,16$ and much higher than that used in Ref. 9. In most conditions it seems to be the third-order dispersion that limits the shortening of the pulses, and a more detailed study is needed in order to know what are the major sources of third-order dispersion in the laser cavity.

The results presented here can be compared with existing results on soliton propagation near zero dispersion..$^{14}$ Wen and $\mathrm{Chi}^{14}$ recently studied the propagation of a soliton with a central wavelength in the neighborhood of zero dispersion. They found that a wave radiates out from the pulse. The spectrum of this wave has a sharp peak at the wavelength $\omega$ such that $\omega-\omega_{0}=3 k_{0}^{\prime \prime} / k_{0}^{\prime \prime \prime}$. They have computed the energy ratio of the radiated wave with respect to the initial soliton energy. They have found that almost no energy $\left(<10^{-2}\right)$ is radiated for a ratio $k_{0}^{\prime \prime \prime} / k_{0}^{\prime \prime} \tau$, where $\tau$ is the pulse width, lower than $10^{-1}$. That means that a spectral resonance is obtained only when the effects of the second- and third-order dispersion terms are of the same magnitude. We observed the same progressive decrease of the resonance peak energy when the GVD increases (Fig. 1).

Nevertheless there is a important difference between our experiment and the analysis of Wen and Chi. In their study they found that the wave radiated out of the soliton progressively spreads because of dispersion. As the velocity of the radiated wave and the soliton are not the same, after a long propagation distance this broad wing should extend a large distance from the soliton itself. In the laser the permanent regime corresponds to an infinite propagation, but the energy of the resonance peak is concentrated in a temporal window of approximately 1 psec around the pulse. We think that the energy that is radiated too far from the main soliton is progressively absorbed by the DODCI and finally disappears. However, at the same time the gain regenerates the energy in the resonance peak even if it continuously spreads.

In summary, we have studied the propagation of pulses near zero GVD in a femtosecond dye laser. We have found that a part of the pulse energy is radiated and trapped in a spectral resonance. The wavelength of this resonance corresponds to the point at which the group velocity is the same as the pulse group velocity. By studying the evolution of the resonance wavelength with respect to the intracavity GVD, we have obtained a value for the third-order dispersion of the laser cavity. This dispersion is found to be of the same order of magnitıde as GVD for pulses shorter than $50 \mathrm{fsec}$. We thus think that the minimal pulse duration achieved in a CPM laser is not currently limited by the laser bandwidth but by high-order dispersion effects.

The authors thank G. R. Boyer for valuable discussions and useful suggestions.

P. Georges is also with Ecole Polytechnique Féminine, 3 bis rue Lakanal, 92230 Sceaux, France.

\section{References}

1. R. L. Fork, B. J. Green, and C. V. Shank, Appl. Phys. Lett. 41, 671 (1981).

2. J. A. Valdmanis and R. L. Fork, IEEE J. Quantum Electron. QE-22, 112 (1986).

3. J. C. Diels, W. Dietel, J. J. Fontaine, W. Rudolph, and B. Wilhelmi, J. Opt. Soc. Am. B 2, 680 (1985).

4. M. Avramopoulos, P. M. W. French, J. A. R. Williams, G. M. C. New, and J. R. Taylor, IEEE J. Quantum Electron. 24, 1884 (1988).

5. V. Petrov, W. Rudolph, U. Stamm, and B. Wilhelmi, Phys. Rev. A 40, 1474 (1989).

6. F. Salin, P. Grangier, G. Roger, and A. Brun, Phys. Rev. Lett. 56, 1132 (1986).

7. F. Salin, P. Grangier, G. Roger, and A. Brun, Phys. Rev. Lett. 60, 569 (1988).

8. F. Salin, P. Grangier, P. Georges, and A. Brun, in Ultrafast Phenomena, Vol. 6 of OSA Technical Digest Series (Optical Society of America, Washington, D.C., 1990), p. 187.

9. M. R. X. de Barros, R. S. Miranda, and C. H. Brito Cruz, Opt. Lett. 15, 127 (1990).

10. R. L. Fork, O. E. Martinez, and J. P. Gordon, Opt. Lett. 9, 150 (1984).

11. A. S. Gouveia-Neto, M. E. Faldon, and J. R. Taylor, Opt. Commun. 69, 173 (1988).

12. F. W. Wise, I. A. Walmsley, and C. L. Tang, Opt. Lett. 13, 129 (1988).

13. G. R. Boyer and X. F. Carlotti, Phys. Rev. A 38, 5140 (1988).

14. S. Wen and S. Chi, Opt. Quantum Electron. 21, 335 (1989).

15. P. K. A. Wai, C. R. Menyuk, Y. C. Lee, and H. H. Chen, Opt. Lett. 11, 464 (1986).

16. W. L. Nighan, Jr., T. Gong, and P. M. Fauchet, IEEE J. Quantum Electron. 25, 2476 (1989). 